which combine together so far as lies in their power to bar all progress, to crush scientific investigation and research, and to wreck every measure which the medical profession as a body, with but few exceptions, demand as essential for the prevention and extermination of some of the most grave and terrible diseases which afflict the human race. Many of our most bitter opponents occupy high social positions and they may be, so far as I know, in their owa spheres well-informed and clever people. They may even have acquired a smattering of physiology, or dabbled in medical works, and the vague and superficial knowledge they have thus obtained makes them conceited enough to imagine that they are thoroughly qualified to give a dogmatic opinion upon every conceivable subject relating to health and disease, as they are indeed quite prepared to take charge of the affairs of the worid in general. But the great mass of opposition which we have to face consists in a combination, in various forms and degrees, of unreasoning prejudice, personal interests which brook no interference, sheer faddism or fanaticism, misguided sentiment, downright ignorance, and consummate impudence and conceit. When any of these obstructive forces are associated with power for active mischief, be it but that of a chairman of a board of guardians, the results are very likely to be most injurious or even disastrous to the cause of humanity. I repeat, therefore, that in face of these embarrassments we must be ever wary and careful as to our sayings and doings, aroiding everything that can rouse needless opposition or excessive friction, and giving no occasion for those whom we are compelled by their conduct to regard as our enemies to gain any advantage over us or to cast ridicule upon our claims.

In bringing this address to a close may I be permitted to refer briefly and comprehensively to what I conceive to be the real objects which such an authoritative and august gathering as an International Medical Congress should have in view and what practical purposes it is intended to serve? Surely it must never be regarded as a mere passing event which leaves behind no permanent impression or result. In general terms a congress of this nature may be described as intended to promote those aims which we as a profession individually and collectively always keep steadily before us notwithstanding our differences of method and in spite of all opposition and difficulties, and by mutual instruction, help and encouragement to render each one of us better fitted to carry out efficiently and intelligently the high functions and noble duties of our calling. And what are they? First and foremost comes the formidable task of diminishing mortality, of preserving and saving life, and prolonging its duration, so far as this purpose is consistent with the fitness of things. As a matter of fact, we instinctively do our atmost under every possible circumstance to avert death, and there is no more sad or trying ordeal for the practitioner of medicine to pass through than to see a young and promising life, or one in the full prime and vigour of manhood, engaged not uncommonly in active and useful work, and, alas, but too often the family "bread-winner," cut off by some dire disease which defies his best skill and most devoted efforts. Our surgicul colleagnes have a great advantage over us poor physicians in this matter and I frequently envy them when $I$ think of the thrill of satisfaction and pleasure which the skilful operator must feel, especially at the present day, when he calls to mind the lives he has personally saved. But the mere addition of two or three years to the average duration of existence, or the prolongation of the life of one bowed down by the ravages of time or with constant pain and suffering, does not appeal to me as the supreme duty of the medical profession and I have no sympathy with some of the ideas now in rogue with regard to the attainment of longevity. I hold in my hand a summary of instructions as to how to live to be a hundred! I wonder who wants to spend a hundred years in this weary world, unless it be that conscience makes him afraid of venturing on the next! Other prominent objects which as a profession we are always striving to falfil are the cure of particular diseases ; their prevention, both among individuals and communities ; and their extermination so far as this is practicable. Then I need but mention the wide field that is open before us and the constant call that is made upon our knowledge and skill in our attempts to relieve pain and suffering of every kind. But I venture to think that perhaps the most difficult problem with which we have to cope at the close of this nineteenth century; and one worthy of our supremest efforts, is how to deal with the mass of "morbidity" and "invalidism," the countless "ailments," real or imaginary, call them by what name you like, which crowd upon us on all hands and in every station of life, which render thousands upon thousands anequal to the ordinary arocations and duties of modern existence, which wreck the comfort and happiness of many a home, and which often become the starting point of pernicious habits that are sapping the very foundations of society in many directions, and if not checked must ineritably lead to widespread disaster.

What will be the ultimate practical outcome of the occasion which I have utilised as a text for my address cannot as yet be determined. We may reasonably hope that it has had some influence in creating a kindly and mutually considerate international feeling at a period in the world's history which is fraught with grave issues. Surely, moreover, it cannot fail to leave its mark upon the history of the progress of medicine and surgery and to rromote in no small measure the all-important objects to which I have just referred. At the present moment we cannot shat our eyes to the disquieting fact that the relations between Russia and Great Britain are such as to cause serious anxiety with regard to the course of events even in the immediate fature. We must all earnestly hope, however, that diplomatic statesmanship, aided by mutual consideration and a conciliatory spirit, may be able to guide us safely and successfully through all dangers and difficulties, so that "peace with honour" may continue between these two great empires. Their power for good upon vast masses of people, when acting in concert, it is impossible to over-estimate; while the beneficial results which may be anticipated from their combined efforts in the canse of civilisation and human progress are incalculable. On the other hand, the bare suggestion of war calls up scenes of horror, devastation, and desolation, with inevitable disastrous consequences to both nations, from which even the most hardened and thoughtless must instinctively shrink. Whatever may be the immediate and final results, international and professional, of this memorable cosmopolitan gathering, and whatever may happen in the fature, I believe I am expressing the feelings and sentiments of my fellowcountrymen who were present and took part in its proceedings when I affirm that while time will dim more and more any passing impression of experiences not altogether agreeable which may have occurred to us as individuals until they are absolutely forgotten, there will always remain a vivid mental picture of novel, attractive, and interesting scenes, as well as of manifestations of kindly feeling and generous hospitality, and we shall one and all ever entertain a gratefal and pleasant reminiscence of the Moscow Congress.

\section{THE VIRUS OF VACCINIA AND ITS CULTIVATION.}

By A. F. STANLEY KeNT, M.A. OXoN.

THE identification and culture of the virus of vaccinia has for many years proved a problem of such difficulty as to baffle the endeavours of some of the most brilliant workers in bacteriology. Perhaps this is in some measure owing to the fact that the methods of bacteriology have been employed almost to the exclusion of histology, the idea that histological methods could afford any assistance having occurred apparently to very few. Nevertheless, that all experiments having for their starting point the collection of lymph from the vaccine vesicle would have to contend with the presence of multitudes of extraneous organisms will be evident to anyone who is conversant with the flora of the skin. It is indeed a matter of common knowledge that micrococci of various species-especially the staphylococcus pyogenes albus and staphylococcus pyogenes aureus-besides being present in large numbers on the surface and in the dead superficial cells of the stratum corneum, swarm in the openings of the hair follicles and even penetrate in them to a considerable depth.

It is for this reason that there arises so extreme a diffculty in sterilising the skin of patients abont to undergo surgical operations, and when the object is to obtain lymph from vesicles in the skin itself and to obtain it free from extraneous organisms it is apparent that the task is one 
calling for the exercice of the utmost care. All attempts to identify the virus by ordinary bacteriological means having failed I proposed to investigate the problem by somewhat different methods. It seemed probable that whatever organisms there might be in the vaccine lymph, at all events in the tissues around the vesicle, one might reasonably hope to find the specific virus in a state of comparative purity. Accordingly I undertook the preparation and examination of a large number of sections of the vaccine vesicle and its surrounding tissues. The preparations were made by vacclnating a susceptible animal in a suitable manner, excising the resulting vesicles at stages of their growth varying from a fex hours after inoculation to their latest development, fixing and hardening these specimens by the best methods known to me, and, finally, after sectioning them, subjecting them to several different methods of stain ing. At the same time lymph was collected of different ages and examined bacteriologically. Some short account of the results obtained was communicated to the meeting of the British Medical Association at Bristol in 1894.

Of the extraneous organisms found it is necessary to say very little. Of course, upon the free surface of the sections, and especially in the openings of the bair follicles, there appeared crowds of micrococci. They were very obvious, occurred in clumps and masses of all sizes, and were as easily stained as is usually the case. They occupied not only the surface of the skin but extended also a considerable distance into the hair follicles, occurring as clumps of varying size and in the deeper parts as groups of two, three, or more. In the tissues immediately bordering on the breach of continuity in the skin small numbers of these organisms may sometimes be seen. In the deeper tissues, however-i.e., a short distance from the actual breach in the surface-they are conspicuous by their absence.

Whilst asserting the absence of the extraneous orgarisms I am far from denying the presence of other organisms in the tissues surrounding the vaccine vesicle-indeed, the exact contrary is the case-and I attach much importance to the fact, which I first pointed out in 1894, that there do exist organisms both in the vesicle and also in the surrounding tissues for a considerable distance from the site of the original lesion-organisms which in their morphological characters, their distribution in the tissnes, their relation to cells, and their staining reactions are separated sharply off from the other organisms ordinarily associated with the processes of vaccinia. Morphologically they may be described as smallindeed, exceedingly minute-bacilli, about 1 micron in length and rather less than a half of this in thickness, with rounded ends, and usually occurring in pairs forming diplo-bacill or dumb-bells. Photographs of these organisms were shown at the Bristol meeting of the British Medical Association in 1894. Morphologically, then, they appear to resemble the minute bacilli described by Klein, and independently by Copeman and myself, as occurring in early vaccine lymph, and I take it that the occurrence of these organisms also in the tissues under circumstances immediately to be described lends some probability to the view that they are fundamentally concerned in the causation of the disease. The distribution of these organisms in the tissues is a point of great importance. Unlike the extraneous organisms, they are not present in countless multitudes at the surface of the skin and in the hair follicles Indeed, as has been shown previounly, they are absent from, or present in small numbers only in, lymph taken from vesicles after the fourth or fifth day from inoculation, whilst the extraneous organisms seem actually to increase in number as time goes on.

On the other hand, the bacillus, which for convenience it may be well to call simply "diplo-bacillus vacciniæ," is present in the tissues surrounding the vaccine vesicle, not only soon after inoculation, but even up to the twelfth or fourteenth day and probably even later. It is of interest to note that it does not occur in the tissues as colonies or irregular masses, but is for the most part contained in cells. These cells appear often to be wandering cells ; some, however, are of the nature of fixed corpuscles. As stated above the presence of these organisms in the tissues surrounding the vaccine vesicle may be recognised in specimens taken at all stages of the disease up to about the fourteenth day and the number does not seem to be materially lessened in preparations made at the later dates. Their presence is the more significant when it is remembered that they are present in what is practically a pure culture, for the extraneous organisms, though numerous on the surface, are absent from the deeper tissues affected by the diplo-bacillus vaccinix. The staining reactions of this bacillus are also of interest inasmuch as they afford some explanation of the extreme difficulty encountered in demonstrating its presence. For to stain the bacillus at all was found difficult and this difficulty has undoubtedly stood in the way of its earlier recognition. Most of the ordinary aniline dyes are unsatisfactory for the purpose and it was only by following out a somewhat complicated modification of Gram's method that I was able to stain it with certainty in the tissues. However, that the method is satisfactory is, I think, proved by the photographs which were shown at Bristol in 1894 and by preparations that were there exhibited.

I consider, then, that the morphological characters, the distribution in the tissues, the relation to cells and the staining reactions, together with the fact that cultures containing this organism produce in the calf and in children typical vaccine vesicles-vesicles which in their turn contain the same virus-sharply separate this diplo-bacillus off from the other so-called extraneous organisms and give support to the view that here at last we have before us the true germ of vaccinia. There are additional c unsiderations which lead to the same conclusion. As will be remembered, a statement has been made that the peculiar minute bacilli described as occurring in early lymph are absent or nearly absent from lymph taken at a later date. In connexion with this it is significant that both Klein and $I^{1}$ have described in these organisms bedies which have the appearance of spores and have noticed the possible explanation by this circumstance of the permanence of vaccine lymph when preserved dry upon points. Is is further to be noted that although the bacilli appear to be absent, or nearly absent, from mature Jymph this is by no means the case with the tissues, the bacilli being recognisable in these until quite a late stage of the disease.

It is of course quite possible that the different conditions obtaining in the two situations are sufficient to determine on the one hand the formation of spores with subsequent or simultaneous breaking up of the bacilli and on the other a persistence of the ordinary form. Nevertheless, it appeared to me to be probable that in the case of ly $\mathrm{mph}$ stored in contact with glycerine some development of the spores, supposing such to exist, might reasonably be looked for and this appeared the more probable from the fact, observed by many, of the great extent to which it is possible to dilute lymph with glycerine without injuring its power, and still more from the statement that ly $\mathrm{mph}$ when so diluted and stored actually increases in virulence. I have accordingly examined a number of samples of glycerinated calf vaccine $1 \mathrm{Kmph}$ and by the special methods of staining above alluded to I have been able to satisfy myself that whatever may be the case with late and mature lymph, in glycerinated lymph which has been kept for a few weeks the diplo-bacillus vacciniæ is undoubtedly present. One explanation may, of course, be that in addition to the lymph itself a certain quantity of resicle pulp would ba used in the preparation of the glycerinated lymph and the bacilli may have come from this, but in most of the samples examined the solid material taken was reduced to a minimum and therefore this source of error was much lessened.

Another thing which tends to strengthen belief in the specific nature of the diplo-bacillus is the fact that, as briefly noted in a joint communication by Dr. Copeman and myself to the Bristol meeting of the British Medical Association in 1894 and more fully described by Dr. Copeman in the following year in London, a method was devised whereby it was found possible to cultivate an organism morphologically identical with the above and to produce by the inoculation of such cultures upon calves vesicles which very closely resembled those of ordinary vaccinia. And, further, I have shown that in vesicles produced by inoculation of cultures the same organism exists and has the same relative distribution as regards cells and extraneous organisms as I have described as characteristic of the diplo-bacillus vaccinix.

It was, however, very desirable that in addition to the cultures made in eggs other media of a more convenient character and capable of being solidified should be devised. I had already made very numerous experiments with all the ordinary culture media and with modifications of these with out attaining any satisfactory result. Early in 1895, however, I was more fortunate and at the meeting of the British Medical Association held in London in that year I was able to report that some measure of success had been obtained.

1 Brit. Med. Jour., Sept. 22nd, 1894. 
My experiments were condacted upon the supposition that it should be possible to prepare some special mixture of albumen and glycerine so proportioned that whilst the former would afford nourishment and encourage the growth of the vaccine virus the latter would prevent the growth of extraneous organisms and so do away with the necessity of sterilisation by heat.

The following is a typical experiment copied from my notebook: "April 18th, 1895: Lymph was collected from the calf at the National Vaccine Establishment in Lamb's Conduit-street. It was inoculated upon a culture medium consisting of egg albumen with the addition of 10 per cent. of pure glycerine and incubated at $37^{\circ} \mathrm{C}$." Five days after inoculation I have a note to the effect that there are many white colonies floating in the fluid, together with a growth which I compared with cotton wool. This cotton-wool-like growth I believe, for reasons which I shall presently set forth, to be the vegetative form of the diplo-bacillus vaccinix. At the same time that the above cultures were made I also inoculated with vaccine lymph tubes of the same culture medium which had been solidified by heat. The note here is that colonies, orange and yellow, are present. There was also up in the surface of the culture medium a thin film.like growth which I took to be the expression on the solid medium of the formation of the cotton-wool-like growth in the liquid. The coloured colonies consisted of staphylococci. Experiments were also carried out with media containing 15 per cent of glycerine, and in these the liquid tubes showed fewer of the white colonies bat more luxuriant cotton. wool-like growth, and on the solid form the orange colonies were usually absent whilst the filmy growth was more pronounced. Finally, when the medium was made up with 20 per cent. or more of glycerine, the liquid tubes showed no colonies whilst the cotton.wool.like growth was well marked. 'These results were briefly alluded to at the meeting of the British Medical Association held in London in 1895 .

On first observing the above-described cotton-wool-like appearance in the culture tubes I was of opinion that it represented a slow and incomplete coagulation or precipita tion of a portion of the albumen contained in the medium such precipitation being due probably partly to the action of the glycerine and partly to the dilution combined with the incubation at $37^{\circ} \mathrm{C}$. As is well known, egg albumen is a complex mixture and contains several proteids which coagulate at different temperatures; moreover, the exact temperature at which coagulation takes place is determined by the concentration of the solution and the quantity of salts present. The coagulation temperature is generally given as from $70^{\circ}$ to $73^{\circ} \mathrm{C}$. . but Gautier states ${ }^{2}$ that coagula may also be obtained at $54^{\circ}$ and $63^{\circ}$, whilst accord. ing to Corin and Berard, ${ }^{3}$ by applying the method of fractional heat ccagulation to filtered white of egg, coagula may be obtained at $575^{\circ}, 67^{\circ}, 72^{\circ}, 76^{\circ}$, and $82^{\circ}$, the first two being due to globulins, the others to albumins. It seemed probable, then, that by mixing egg albumen with gly cerine and subjecting it to incubation some amount of precipitation of proteid might be productd and this was rendered more likely by the experiments of W. Ramsden, who showed that by a prolonged action of heat a precipitation of albuminous fluids might be brought about at a temperature considerably below that generally assigned as specific for the body under consideration.

Accordingly I made numerous control experiments by mixing with egg albumen varying quantities of glycerine and submitting the resulting mixtures to similar conditions of incubation as obtained in the case of the culture tubes. Treated in this way it was found that in the case of tubes containing an addition of 33 per cent. of glycerine after a short time a certain amount of a woolly material appeared as clumps and cloudy patches in the fluid. It appeared, however, to consist of a definite solid substarce suspended in a bright and transparent liquid and no general opalescence or cloudiness of the fluid was produced. In the case of cultures, on the other hand, the solid portion was much more finely divided and produced a definite opalescence in the liquid. Larger flakes of a peculiar semi-transparent character were also present, and at the npper edge of the fluid there was often observed a filmlike portion of growth. In tubes containing a less proportionate amount of glycerine-viz., about 16 per cent.-and in

2 Chimie appliquée à la Physiologie, \&c., Tome i. (1874), p. 242. p. 170 . which the albumen was about 33 per cent., a greater degree of opalescence was produced, but no increase in the cloudiness was observed to occur after the first few days, whilst in the case of similar tabes inoculated with vaccinia the opalescence was much greater, continned to increase and was accompanied by the formation of a flaky semi-transparent material, especially at the upper part of the tube where the fluid was in contact with air. In solid media it is of course less easy to make ont the exact characters of the growth, in consf quence of the physical condition of the nutrient material. It is, however, possible to ascertain that in its general characters the growth corresponds to the filmlike material seen at the upper part of tubes containing liquid culture material.

Having thus succeeded in obtaining a growth in special culture media inoculated with vaccine lymph I next experimented with similar media incculated with lymph which by admixture with glycerine and subsequent storage had been freed from extraneous organiems. These experiments gave results indistinguishable from those above described, with the exception that in no case did coloured colonies appear. Moreover, when cover glass preparations had been made and appropriately stained microscopical examination revealed in every case the presence of groups of organisms morphologically indistinguisbable from that above described as diplo-bacillus vacciniæ. Further, in the majority of the experiments they were the only organisms present, but in a few cases they were accompanied by extraneous germs. These cultures have been used for the inoculation of calves and have given results which tend to support the view of the specific nature of the bacil'us.

It may rerhaps be suggested that the finding of the organisms in the cultures is no proof of their multiplication in the artificicl medium, since the individuals observed microscopically may have been some of those present in the original lymph used for establishing the culture. In answer to this objection I need orly say that th e cultures have been carried on from tube to tube and also the quantity of lymph

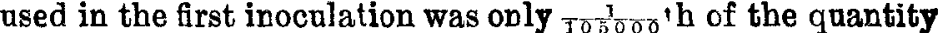
of fluid with which it was mixed. So that although in the tubes of the first generation cover-glass preparations might conceivably reveal the presence of an occasional bacillus, the occurrence of numerous groups would be most unlikely and would approach impossibility in the case of subsequent tube-generations bad no multiplication taken place meanwhile. As mentioned previously, in a communication to the British Medical Association in 1894 it was sta'ed that cultures of an organis morphologically indistinguishable from the above, but obtained from variolous crusts, when inoculated upon calves produced phenomena which up to the fourth day were similar to those produced by normal lymph, whilst in 1895 Dr. Copeman communicated to the association the further fact that by passing such cultures through two successive calves a lymph was obtained which produced typical vaccinia in a child. The cultures with which these results were obtained were fully described by Dr. Copeman in 1895. The medium used was the hen's egg, the yelk and white being well mixed together and subsequently inoculated with variolous material. Certain disadvantages were found to result from the use of such nutrient material. Transparency was lacking, subcultures failed, and isolation of special organisms by plate cultivations was not practicable. It was whilst endeavouring to improve upon these early attempts that $I$ hit upon the plan of using the egg-white apart from the yelk and mixed with safficient glycerine to inhibit the growth of extraneous organisms.

The result has been so far successful that $I$ am $n: w$ able to place the diplo-bacillus vaccinim under i ir nms ances such that its muliplication is apparen prepare cultures containing it in a pure state.

James-street, Hyde Fark, W.

Crownhill (Devonshire) Convalescent Home? - The annual meeting of this institution was held on April 30th. The report showed that 265 patients had been admitted during 1897. The financial statement exbibited a balance in hand of $£ 28$. The report mentic ned that the home, which is beautifully situated on the border of Dartmoor, is capable of accommodating 500 patients during the year, but the subscription list is not large enough to permit of this namber being received. 